\title{
Evaluation of the integral methods for the kinetic study of thermally stimulated processes in polymer science
}

L.A. PÉREZ-MAQUEdA ${ }^{*}$, P.E. SÁNCHEZ-JiMÉNEZ AND J.M. CRIADO

Instituto de Ciencia de Materiales de Sevilla. C.S.I.C.-Universidad de Sevilla. Avd. Americo Vespucio 41092 Sevilla. Spain

*Corresponding author. E-mail address: maqueda@cica.es 


\section{Abstract}

This paper reports on the accuracy of the integral methods used for the kinetic analysis of degradation and crystallization of polymers. Integral methods are preferred by many authors over the differential ones because often the experimental data obtained, such as thermal degradation studied by thermogravimetry, are integral and the differentiation of the integral data usually produces an unwilling increase of the noise. A problem of the integral methods is the fact that Arrhenius integral function does not have an exact analytical solution. Thus, several approximated equations have been proposed in literature. Some of these approximations lead to a linear relation between the logarithm of $g(\alpha)$ and a predetermined function of $T$, in such a way that the activation energy can be determined from the slope of the plot of $\ln g(\alpha)$ versus the predetermined $T$ function. The most popular approximations to the Arrhenius integral in polymer science are those of Van Krevelen et al, Horowitz and Metzger, and Coats and Redfern. Although these three approaches where proposed fifty years ago, they are extensively used nowadays and several hundreds of citations to the original papers can be found in recent polymer science publications. Despite their popularity, there are cast doubts on the accuracy of these approximations, because they provide significant deviations in the determination of the actual values of the Arrhenius integral when used for simulating $\alpha$-T plots. Nevertheless, a comprehensive study of the systematic errors in the activation energy calculated from these integral methods is still missing. In this paper a comparative study of the accuracy of the different integral methods is performed. The calculated errors are tested with simulated and experimental results.

Keywords: Kinetics; Activation Energy; Thermal Properties; Teflon 


\section{INTRODUCTION}

Studies of thermal degradation of polymers are a matter of major interest for determining the thermal stability of the polymers. Thermogravimetry (TG) has been extensively used for such studies. Experiments are usually performed under linear heating rate program. Under these conditions, the temperature varies in a wide range of values and the thermal behaviour of the sample can be recorded in a single experiment. In the TG experiment, the recorded magnitude, i.e. mass, is proportional to the extent of the reaction $(\alpha)$. The TG data can be used for obtaining the kinetic parameters of the thermal degradation of the polymer sample. To perform the kinetic study of the integral curve (mass loss versus temperature) directly obtained from thermogravimetric analysis, it is necessary to use an integral analysis method. A limitation of the integral methods of kinetic analysis is that the temperature integral does not have an exact analytical solution for linear heating rate program [1]. In literature, different approaches have been reported for this integral, such as rational approximations that allow accuracies better than $10^{-8} \%$ in the estimation of the temperature integral [2]. Other approximations to the temperature integral lead to linear correlations between the logarithm of the integral function describing the mechanism of the process and a function of temperature, in such a way that the activation energy is obtained from the slope. Among these latter approximations, the most commonly used in studies of thermal degradation of polymers (see for example references [3-6] for some recent papers) are those of Van Krevelen et al. [7], Horowitz and Metzger [8], and Coats and Redfern [9, 10]. In fact, for the original papers of Coats and Redfern [9, 10], Horowitz and Metzger [8], and Van Krevelen et al. [7] we have found about 400 citations in polymer papers published only in the last five years (from 1999 to nowadays, this information was obtained from ISI 
Web of Science data base). Nevertheless, there are still some doubts about their accuracy for determining the kinetic parameters [1, 11-14] because their lack of accuracy in the estimation of the temperature integral. However, the most common application of these approximations is the determination of the activation energy and not the computation of the temperature integral. In this paper we intend to perform a comparative study of the accuracy of such approximation to the temperature integral for the determination of the activation energy. The calculated errors are checked with simulated and experimental curves. 


\section{THEORY}

The general equation that describes the reaction rate for the thermal degradation of polymers can be written as follows:

$$
\frac{d \alpha}{d t}=A e^{-E / R T} f(\alpha)
$$

that after taking logarithms can be rearranged in the following form

$$
\ln \frac{(d \alpha / d t)}{f(\alpha)}=\ln A-\frac{E}{R T}
$$

where $t$ is the reaction time, $\alpha$ is the extent of reaction, $A$ the preexponential factor of Arrhenius, $E$ the activation energy, $R$ the gas constant, and $f(\alpha)$ is a function depending on the reaction mechanism.

Eqn. 1 is a constitutive equation that must be accomplished whatever would be the thermal pathway used for achieving a particular value of T- $\alpha-\mathrm{d} \alpha / \mathrm{dt}$ [15-18].Thus, the activation energy of a degradation reaction recorded under linear heating rate would be obtained from the plot of the left hand side of Eq. (2) as a function of $1 / \mathrm{T}$.

Eq. (1) cannot be directly integrated under rising temperature conditions unless the the temperature were a known function of the time. If the degradation of polymers is recorded under a linear heating rate $\beta=d T / d t$, Eq. (1) would also be written in the form

$$
\frac{d \alpha}{d T}=\frac{A}{\beta} e^{-E / R T} f(\alpha)
$$

The integration of Eq. (2) leads to

$$
g(\alpha)=\int_{0}^{\alpha} \frac{d \alpha}{f(\alpha)}=\frac{A}{\beta} \int_{0}^{T} e^{-\frac{E}{R T}} d T
$$

or

$$
g(\alpha)=\frac{A}{\beta} I
$$


where $I$ stands for the integral of the Arrhenius equation. Eqs. (3a) and (3b) allow the direct analysis of the experimental data obtained from the measurement of the evolution of an integral magnitude, such as the mass loss recorded in a thermobalance as a function of the temperature under a linear heating program.

The integral of the Arrhenius equation does not have an exact solution and therefore this expression cannot be expressed in a closed form. Several approaches have been proposed in literature for the integral of the Arrhenius equation in order to determine the activation energy from experimental integer data. As it was mentioned above, in polymer science, the most commonly used approaches are those of Van Krevelen et al [7], Horowitz and Metzger [8], and Coats and Redfern [9, 10]; the corresponding approximated Arrhenius integral functions, $I_{a}$, are included in Table 1. The use of these approximations implies using the following equation:

$$
g(\alpha)=\frac{A}{\beta} I_{a}
$$

instead of the exact Eq. (3b) for calculating the activation energy.

In general, the approximations to the temperature integral have been chosen in such a way that the plot of the logarithm of the corresponding $g(\alpha)$ function versus a function of temperature $(f(T))$ lead to a straight line from whose slope is determined the activation energy:

$$
\frac{d \ln (g(\alpha))}{d f(T)}=f\left(E_{a}\right)
$$

The corresponding $f(T)$ and $f\left(E_{a}\right)$ functions listed in Table 1 depend on the considered approximation to the Arrhenius integral and the subindex $a$ stands for approximated.

The relative error $\varepsilon$ of the activation energy $\left(E_{a}\right)$ calculated by the different approximated equations can be defined by the following equation: 


$$
\varepsilon \%=\frac{E_{a}-E}{E} 100=\left(\frac{E_{a}}{E}-1\right) 100
$$

By differentiating Eq. (3b) with regards to $f(T)$ we get

$$
\frac{d \ln g(\alpha)}{d f(T)}=\frac{d \ln I}{d f(T)}
$$

By combining eq. (5) and (7) results

$$
\frac{d \ln I}{d f(T)}=f\left(E_{a}\right)
$$

By introducing the variable $x=E / R T$, it is shown that the derivative of $f(T)$ is connected with the derivative of $x$ through the expression:

$$
d f(T)=\frac{R}{E} f^{\prime}(T) \cdot T^{2} d x
$$

Thus, from Eqs. (8) and (9), we get

$$
-\frac{d \ln I}{d x}=\frac{R}{E} T^{2} f^{\prime}(T) f\left(E_{a}\right)
$$

The value of $E_{a} / E$ as a function of $x$ and $d \ln I / d x$ can be directly obtained from Eq. (10) after the substitution of the $f\left(E_{a}\right)$ and $f^{\prime}(T)$ corresponding to the different approximated equations tabulated in Table 1. Finally, after substituting the expressions for $E_{a} / E$ into Eq. (6), the errors functions included in Table 2 were obtained for the Van Krevelen et al [7], Horowitz and Metzger [8] and Coats and Redfern,[9, 10] approaches, respectively.

The error expressions shown in Table 2 indicate that the errors in the activation energy depend on the values of $x=E / R T$ for the three approximated equations here analysed. The values of $\varepsilon \%$ tabulated in Table 3 for the different approaches have been calculated as a function of $E / R T$ after calculating the corresponding values of $d \ln I / d x$ 
by numerical methods using the MathCad software with a tolerance better than $10^{-9}$. It is clear from this table than the errors significantly change as a function of $x$. Thus, the larger the value of $x$, the smaller the error; in the limit, for $x=\infty$, the error is zero. The approximation of Coats and Redfern is the one that leads to the smallest errors in the determination of the activation energy, followed by that of Van Krevelen et al., while that of Horowitz and Metzger is the lest accurate one in terms of determining activation energies.

\section{EXPERIMENTAL}

A commercial polytetrafluoroethylene (Teflon) sample supplied by Aldrich was used. Thermogravimetric experiments were performed under linear heating rate conditions $\left(\beta=5 \mathrm{~K} \mathrm{~min}^{-1}\right)$ and flow of helium $\left(100 \mathrm{~cm}^{3} \mathrm{~min}^{-1}\right)$ in a Stanton Redcroft TG-770 thermobalance.

\section{CHECK OF THE ERRORS WITH SYMULATED AND EXPERIMENTAL} CURVES

To check the values of the errors tabulated in Table 3, a set of two curves (Fig. 1) have been simulated for linear heating rate conditions (heating rate $\beta=10 \mathrm{~K} \mathrm{~min}^{-1}$ ), F1 kinetic model and the kinetic parameters included in the figure caption. The simulations have been performed by solving eq. (1) by the Runge-Kutta method using the Mathcad software and a tolerance (precision in the calculus) of $10^{-9}$ The values of the activation energies $\left(E_{a}\right)$ and errors $(\varepsilon \%)$ obtained of the analysis of the simulated curves included in Figs $1 \mathrm{a}$ and $1 \mathrm{~b}$ by means of the different integral methods are included in Table 4 . The resulting values of $\varepsilon \%$ are consistent with those tabulated in Table 3. The small deviations observed are due to the fact that the errors tabulated in 
Table 3 have been calculated for determined values of $x$, while for the simulated curves $T$ varies during the experiment and the values of $x$ are not constant along the entire simulated curve. On the other hand, it is noteworthy to point out that the activation energies obtained from the Van Krevelen et al. and Horowitz and Metzger methods would be very sensitive to the selected reference temperature.

Figure 2 shows the thermal decomposition of the Teflon sample under linear heating rate conditions. This curve has been analyzed by means of the differential and integral methods by assuming an F1 kinetic model. For the integral analysis, the three different approaches analyzed here have been used. Table 5 includes the resulting values of the activation energy, correlation coefficient and errors in the activation energy, as calculated by assuming the value of $E$ obtained from the differential method as the correct one because no approximation is used for deriving Eqns. (1) and (2). Using the kinetic parameter obtained of the differential method, the Teflon decomposition curve has been reconstructed (line in Fig 2). The agreement between the experimental (circles) and simulated (line) curve in Fig. 2 is excellent. For the different kinetic analysis methods included in Table 5, the correlation coefficients are very high, while the values of the corresponding activation energy slightly differ. The errors in the values of $E_{a}$ are consistent with those expected for a value of $x \approx 40$ and tabulated in Table 3.

Although it is not common to find in literature papers where the thermal degradation of polymers is analyzed simultaneously by the different integral equations analyzed here, we have found some recent papers published in Polymer where the same experimental results are systematically analyzed by a series of different integral method. Thus, in the paper by Nuñez et al. [3], the authors report on the decomposition of the epoxy system $\operatorname{BADGE}(\mathrm{n}=0) / 1,2 \mathrm{DCH}$, and perform an exhaustive kinetic analysis 
using the different kinetic integral approaches. Thus, for the $5-15 \mathrm{~K} \mathrm{~min}^{-1}$ experiments, data are fitted by an A4 kinetic model. The resulting values of the activation energy obtained using the Coats and Redfern method range from 142.1 to $144.4 \mathrm{~kJ} \mathrm{~mol}^{-1}$, while those $E_{a}$ obtained using the Van Krevelen et al. and Horowitz and Metzger methods range from 148.8 to $151.4 \mathrm{~kJ} \mathrm{~mol}^{-1}$ and from 154.3 to $157.7 \mathrm{~kJ} \mathrm{~mol}^{-1}$, respectively. These values clearly indicate that the $E_{a}$ values obtained from the different approaches slightly differ. Considering that the value of $x$ for this reaction is about 44 , the $E_{a}$ obtained by the Coats and Redfern method should have an insignificant error (Table 3) while the $E_{a}$ obtained by the other two methods should have a positive error larger than $2 \%$. Thus, the differences observed for the activation energies obtained by Nuñez et al. are consistent with the errors in the activation energy included in Table 3 . In another paper, Chang et al. [5] report on the kinetics of the dehydration and decomposition of the copolymer PMPS-PVI. These authors use the Van Krevelen et al and Coats-Redfern methods. The calculated values of activation energy for both processes are presented in a chart where it is clear that the values obtained by the Van Krevelen et al method are in both cases higher than those obtained by the Coats and Redfern method, as would be expected from Table 3, where it is shown that the $E_{a}$ obtained by the Coats-Redfern method has a small negative error while that obtained by the Van Krevelen et al method is subjected to a larger positive error.

In conclusion, it has been observed that the different approximations to the Arrhenius integral lead to systematic errors in the values of the activation energy determined by means of these equations. The errors are tabulated in Table 3. Nevertheless, the resulting errors are only significant for values of $E / R T$ relatively small, while for large values of $E / R T$ these errors are very small and in the range of the expected experimental errors. It have been also observed that the Coats and Redfern 
approach is preferred over those of Van Krevelen et al. and Horowitz and Metzger because the resulting errors are much smaller for the entire range of $E / R T$. Moreover, the error in the activation energy obtained from the methods of Van Krevelen et al. and Horowitz and Metzger would be influenced by the value of the reference temperature. The values of the errors have been checked by analyzing by the different methods simulated and experimental curves, showing an excellent agreement. Finally, the differences reported in some recent publications for the activation energy values obtained by the different integral methods have been explained in terms of the systematic errors of the integral methods.

\section{REFERENCES}

[1] Flynn JH. Thermochim. Acta 1997;300:83.

[2] Pérez-Maqueda LA, Criado JM. J. Therm. Anal. Calorim. 2000;60:909.

[3] Nunez L, Fraga F, Nunez MR, Villanueva M. Polymer 2000;41:4635.

[4] Wu CS, Liu YL, Hsu KY. Polymer 2003;44:565.

[5] Chang TC, Yeh TF, Yang CW, Hong YS, Wu TR. Polymer 2001;42:8565.

[6] Zvetkov VL. Polymer 2001;42:6687.

[7] van Krevelen DW, van Heerden C, Huntjens FJ. Fuel 1951;30:253.

[8] Horowitz HH, Metzger G. Anal. Chem. 1963;35:1464.

[9] Coats AW, Redfern JP. Nature 1964;201:68.

[10] Coats AW, Redfern JP. Journal of Polymer Science Part B-Polymer Letters $1965 ; 3: 917$.

[11] Flynn JH. Thermochim. Acta 1992;203:519.

[12] Heal GR. Thermochim. Acta 1999;341:69. 
[13] Heal GR. Instrum. Sci. Technol. 1999;27:367.

[14] Wanjun T, Yuwen L, Hen Z, Zhiyong W, Cunxin W. J. Therm. Anal. Cal. $2003 ; 74: 309$.

[15] Criado JM, Pérez Maqueda LA. In: Sorensen OT, Rouquerol J, editors. Sample Controlled Thermal Analysis: Origin, Goals, Multiple Forms, Applications and Future. Dordrecht: Kluwer, 2003. pp. 55.

[16] Criado JM, Perez-Maqueda LA, Gotor FJ, Malek J, Koga N. J. Therm. Anal. Cal. 2003;72:901.

[17] Perez-Maqueda LA, Criado JM, Gotor FJ, Malek J. J. Phys. Chem. A $2002 ; 106: 2862$.

[18] Perez-Maqueda LA, Criado JM, Malek J. J. Non-Cryst. Solids 2003;320:84. 
TABLE 1. $I_{a}$ functions corresponding to the approaches of Van Krevelen, Horowitz and Metzger, and Coats and Redfern to the integral of the Arrhenius equation and their related functions $f(T), f^{\prime}(T)$ and $f\left(E_{a}\right)$ as defined by Eq. (5).

\begin{tabular}{|c|c|c|c|c|}
\hline Approximation & $\boldsymbol{I}_{\boldsymbol{a}}$ & $\boldsymbol{f ( T )}$ & $\boldsymbol{f}^{\prime}(\mathbf{T})$ & $\boldsymbol{f ( \boldsymbol { E } _ { \boldsymbol { a } } )}$ \\
\hline Van Krevelen $^{1}$ & $\left(\frac{0.368}{T_{m}}\right)^{E / R T_{m}}\left(\frac{E_{a}}{R T_{m}}+1\right)^{-1} T^{\left(E / R T_{m}\right)+1}$ & $\ln (T)$ & $1 / T$ & $\frac{E_{a}}{R T_{m}}+1$ \\
\hline Horowitz and $_{\text {Metzger }}^{2}$ & $\frac{R T_{s}^{2}}{E_{a}} \exp \left(-E_{a} / R T_{s}\right) \exp \left(E_{a} \theta / R T_{s}^{2}\right)$ & $\theta$ & 1 & $\frac{E_{a}}{R T_{s}^{2}}$ \\
\hline Coats and & $e^{-E_{a} / R T}$ & $1 / T$ & $-T^{2}$ & $-2 T-\frac{E_{a}}{R}$ \\
\hline Redfern & $\left(E_{a} / R T^{2}\right)$ & & \\
\hline
\end{tabular}

${ }^{1} T_{m}$ is a reference temperature; usually it is the temperature at with reaction rate is a maximum.

${ }^{2} \theta=T-T_{s}$, being $T_{s}$ an arbitrary reference temperature.

TABLE 2. Expressions for the relative errors of the activation energy as calculated from the different approximation of the Arrhenius integral.

\begin{tabular}{|l|l|}
\hline Approximation & Relative error \\
\hline Van Krevelen et al. & $\varepsilon \%=\left(-\left(\frac{\partial \ln (I)}{\partial x}\right)-\frac{1}{x}-1\right) 100$ \\
\hline Horowitz and Metzger & $\varepsilon \%=\left(-\left(\frac{\partial \ln (I)}{\partial x}\right)-1\right) 100$ \\
\hline Coats and Redfern & $\varepsilon \%=\left(-\frac{\partial \ln (I)}{\partial x}-\frac{2}{x}-1\right) 100$ \\
\hline
\end{tabular}


TABLE 3. Values of the relative error $(\varepsilon \%)$ for the activation energy calculated by means of the Van Krevelen, Horowitz and Metzger, and Coats and Redfern equation as a function of the parameter $x=(E / R T)$.

\begin{tabular}{|r|c|c|c|}
\hline \multicolumn{1}{|c|}{$\boldsymbol{x}$} & Van Krevelen & Horowitz Metzger & Coats Redfern \\
\hline $\mathbf{2}$ & 30.28 & 80.28 & -19.72 \\
\hline $\mathbf{5}$ & 15.23 & 35.23 & -4.76 \\
\hline $\mathbf{1 0}$ & 8.53 & 18.53 & -1.47 \\
\hline $\mathbf{2 0}$ & 4.58 & 9.58 & -0.42 \\
\hline $\mathbf{3 0}$ & 3.13 & 6.47 & -0.20 \\
\hline $\mathbf{5 0}$ & 1.92 & 3.92 & -0.07 \\
\hline $\mathbf{1 0 0}$ & 0.98 & 1.98 & -0.02 \\
\hline$\infty$ & 0 & 0 & 0 \\
\hline
\end{tabular}


TABLE 4. Values of the activation energies $\left(E_{a}\right)$ and errors $(\varepsilon \%)$ obtained of the analysis of the simulated curves included in Figs $1 \mathrm{a}$ and $1 \mathrm{~b}$ by means of different integral methods

\begin{tabular}{|l|c|c|c|c|}
\cline { 2 - 5 } \multicolumn{1}{c|}{} & \multicolumn{2}{c|}{$\begin{array}{c}\text { Simulated curve Fig. 1a } \\
(x \approx 5) *\end{array}$} & \multicolumn{2}{c|}{$\begin{array}{c}\text { Simulated curve Fig. 1b } \\
(x \approx 20) *\end{array}$} \\
\cline { 2 - 5 } \multicolumn{1}{c|}{} & $E_{a}\left(\mathrm{~kJ} \mathrm{~mol}^{-1}\right)$ & $\varepsilon \%$ & $E_{a}\left(\mathrm{~kJ} \mathrm{~mol}^{-1}\right)$ & $\varepsilon \%$ \\
\hline Van Krevelen et al. & 29.9 & 19.9 & 106.9 & 6.9 \\
\hline Horowitz Metzger & 35.7 & 42.9 & 114.3 & 14.3 \\
\hline Coats Redfern & 24.0 & -4.0 & 99.6 & -0.4 \\
\hline
\end{tabular}

${ }^{*}$ The value of $\mathrm{x}$ corresponds to the temperature at which $\alpha=0.5\left(x=E_{a} / R T_{0.5}\right)$.

Table 5. Results of the kinetic analysis of the thermal decomposition curve for the Teflon sample by different methods. The relative errors $(\varepsilon \%)$ in the activation energy calculated by means of the approximated integral methods are also included.

\begin{tabular}{|c|c|c|c|}
\hline & $\mathrm{r}$ & $E_{a}\left(\mathrm{~kJ} \cdot \mathrm{mol}^{-1}\right)$ & $\varepsilon^{0}$ \\
\hline Differential & 0.999 & 310.8 & - \\
\hline Coats Redfern & 0.999 & 309.9 & -0.3 \\
\hline Horowitz Metzger & 0.999 & 326.1 & 5.2 \\
\hline Van Krevelen & 0.999 & 319.3 & 2.8 \\
\hline
\end{tabular}




\section{Figure Captions}

Fig. 1. Simulated curves under linear heating rate conditions $\left(\beta=10 \mathrm{~K} \mathrm{~min}^{-1}\right)$, F1 kinetic model: (a) $E=20 \mathrm{~kJ} \mathrm{~mol}^{-1}$ and $A=12 \mathrm{~min}^{-1}$; and (b) $E=100 \mathrm{~kJ} \mathrm{~mol}^{-1}$ and $A=10^{8} \mathrm{~min}^{-1}$

Fig.2. Experimental curve $\left({ }^{\circ}\right)$ for the thermal decomposition of Teflon under linear heating rate conditions $\left(\beta=5 \mathrm{~K} \mathrm{~min}^{-1}\right)$ and flow of helium $\left(100 \mathrm{~cm}^{3} \mathrm{~min}^{-1}\right)$. The solid line represents the curve simulated for an F1 kinetic model and the activation energy obtained from the analysis of the experimental curve by means of the differential method (Table 5). 


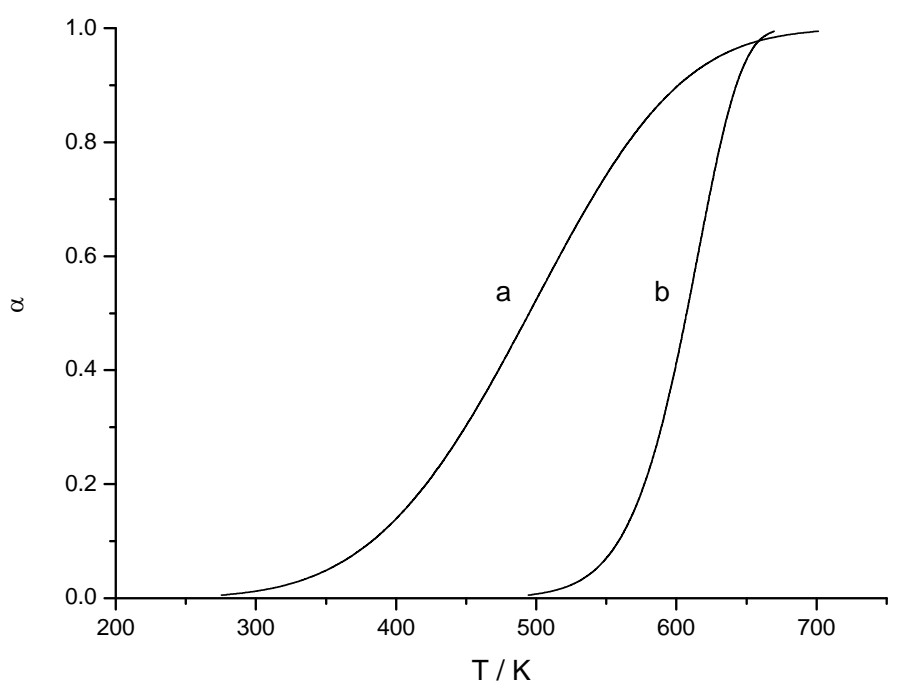

Fig. 1 


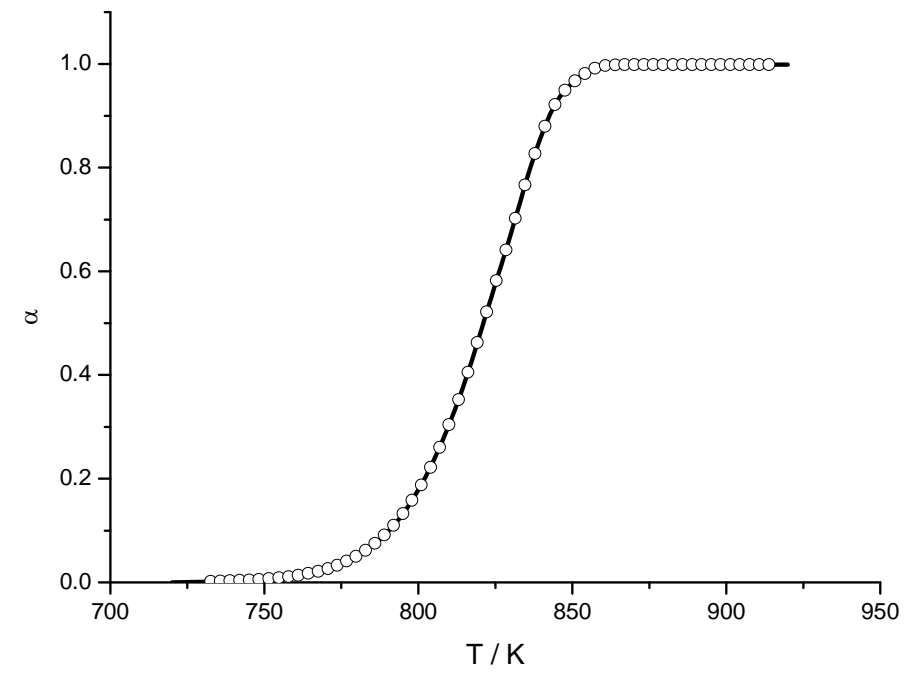

Fig. 2. 